\title{
RECONSTRUCTION OF AN ORBITAL FLOOR FRACTURE IN A 4-YEAR-OLD CHILD
}

\author{
Viktor Chrobok ${ }^{1}$, Arnošt Pellant ${ }^{1}$, Jan Novák ${ }^{2}$, Jan Brožík ${ }^{3}$
}

Regional Hospital Pardubice, University Pardubice, Faculty of Medical Studies, Czech Republic: Department of Otorhinolaryngology, Head and Neck Surgery ${ }^{1}$, Department of Ophthalmology ${ }^{2}$; Charles University in Prague, Faculty of Medicine and University Hospital Hradec Králové, Czech Republic: Department of Diagnostic Radiology 3

Summary: The authors present a case of a 4-year-old girl with an orbital floor fracture. During surgery absorbable collagenous mesh (Pelvicol) was placed between the bone wall and the orbital periosteum. Pelvicol was selected as a natural xenogeneic tissue (porcine dermis) ready for first use in the reconstruction of the orbital floor fracture. The patient has fully recovered without any symptoms.

Key words: Orbital floor fracture; Collagenous mesh; Surgery

\section{Introduction}

The most frequent orbital wall fractures involve the orbital floor. Such fractures can be divided into transmarginal and retromarginal (blowout) fractures. Two theories of retromarginal fracture aetiology have been described: the hydraulic theory and the bone conduction mechanism.

The former refers to a direct force to the globe and its compression (blowout), which increases intraorbital pressure and leads to a fracture of the weakest point of the orbital bone (the orbital floor or the medial orbital wall). The latter suggests a force to the lower orbital rim, which transfers pressure to the orbital floor and results in its fracture (9).

A conservative or a surgical therapeutic approach should be selected according to the extent and localization of the injury and according to the difficulties exhibited by the patients. In addition, retromarginal fractures of the orbital floor in children are unique due to the pathology involved in the orbital bone injury and due to a distinctive surgical approach.

The report presents a fracture of the orbital floor in a child. Furthermore, therapeutical options in retromarginal fractures of the orbital floor are discussed.

\section{Case Report}

A 4-year-old girl was running in a garden and hit her face against an agricultural machine. Subsequently, she started to bleed from her nose, and a haematoma of her left lower eyelid and her left cheek developed. She did not have any period of unconsciousness. A CT examination of the orbits and the paranasal sinuses in the coronal plane revealed a retromarginal fracture of the orbital floor with large soft tissue herniation into the maxillary antrum (Fig. 1) with no ocular muscle restriction. A consulting ophthalmologist did not find any visual acuity impairment. Furthermore, movements of the eyeballs were not restricted, and the child did not report diplopia. Oedema subsided on the 6 th day after the injury, and surgery was performed. The indication for surgery was a large soft tissue herniation in the maxillary sinus. Bone fragments of the orbital floor and a tear of the perforated orbital periosteum were found after performing a transconjuctival incision combined with a lateral canthotomy. After lifting the soft orbital tissues and the orbital periosteum from the maxillary antrum, a partly absorbable collagenous mesh (Pelvicol) was placed between the bone wall and the orbital periosteum to provide support. A forced duction test prior to and after the reduction proved an absence of extraocular muscle restriction. Next, the incision was sutured. After the surgery the child made an uneventful recovery, her visual acuity was not impaired, her eyeball movements were not restricted, and diplopia was not present. A follow-up MRI examination of the orbits and the paranasal sinuses one month after the surgery demonstrated the collagenous mesh and the orbital soft tissues in a good position without any signs of dislocation into the maxillary antrum (Fig. 2).

\section{Discussion}

The region medial to the infraorbital neurovascular bundle, approximately $10 \mathrm{~mm}$ posterior to the orbital margin, is a typical location of retromarginal (blowout) fractu- 
res of the orbital floor. Complications and difficulties for the patient usually depend on the size of the upper wall of the maxillary antrum, and on the localization, the type and extent of the fracture. These factors influence the grade and character of the orbital soft tissues. Small fractures could be asymptomatic or they could be complicated by extraocular muscle restriction (most frequently, by restriction of the inferior rectus muscle) with a subsequent eyeball movement disorder. On the other hand, large fractures of the orbital floor result in enophthalmos (2). The prevention of

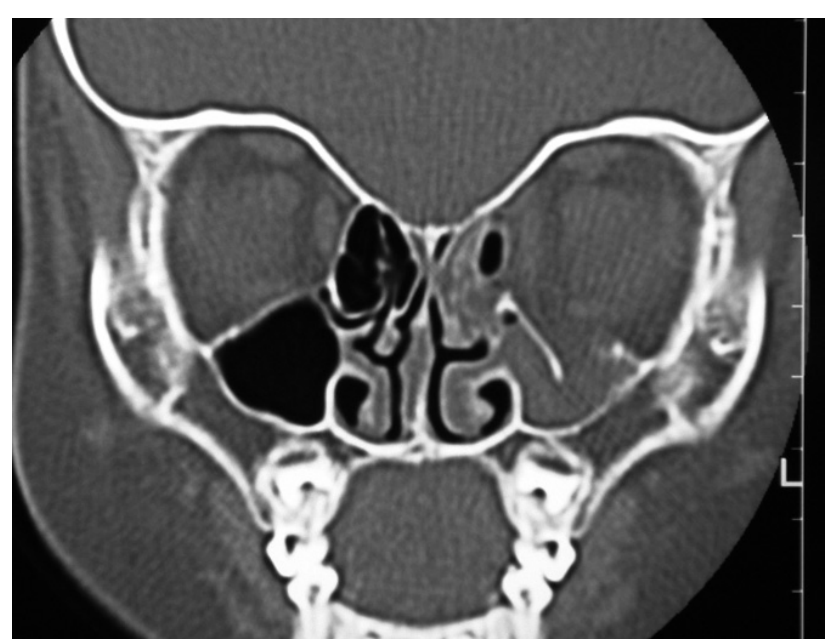

Fig. 1: A CT examination of the orbits and the paranasal sinuses in the coronal plane revealed a retromarginal fracture of the orbital floor with large soft tissue herniation into the left maxillary antrum without restriction of ocular muscles. The edema or hematoma is in the left ethmoid cells.

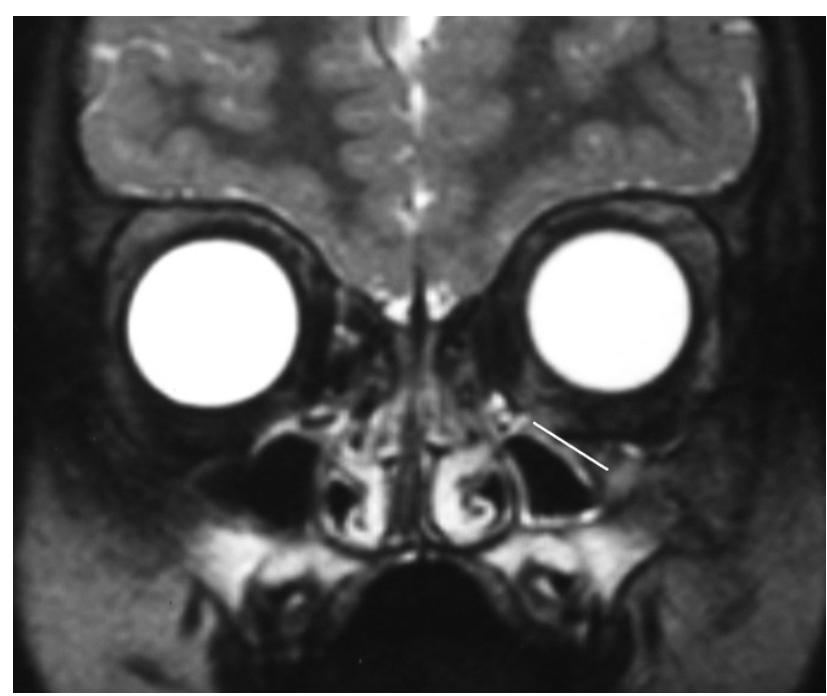

Fig. 2: An MRI examination of orbits and paranasal sinuses one month after the surgery demonstrated the collagenous mesh (white line) and the orbital soft tissues in a good position without signs of dislocation into the left maxillary antrum. enophtalmos was the indication for surgery in our case. Extraocular muscle restriction or a considerable dislocation of the globe causes diplopia. To asses the extent of a fracture and herniation of the soft orbital tissues into the maxillary antrum (12), either a CT examination of the orbits and the paranasal sinuses in the coronal plane (11) or an endoscopy of the antral cavity is used. Following these examinations, a decision is made whether surgical intervention and reduction should be performed. The goals of the orbital floor fracture repair are to release the incarcerated or prolapsed orbital tissue from the fracture defect and to restore correct anatomy.

In children, retromarginal fractures of the orbital floor differ from fractures of the orbital floor in adults $(2,5)$. In adults, it is usual to see mostly extensive comminuted orbital floor fractures with herniation of the orbital contents into the maxillary antrum and with possible subsequent enophthalmos.

The term paediatric facial fractures refers to fractures in children under the age of 10 with an incomplete permanent dentition. Children older than 10 years of age are essentially treated as adults (3). On the other hand, in children with a small maxillary antrum, the bone wall of the orbit is elastic and the orbital periosteum is firm; therefore, they suffer small fractures. Frequently, a fracture of the closed hatch is observed, with a high probability of the inferior rectus muscle extraocular restriction (in 70-85 \% cases). There-

Tab. 1: Diagnostic and therapeutic procedures in orbital floor fractures.

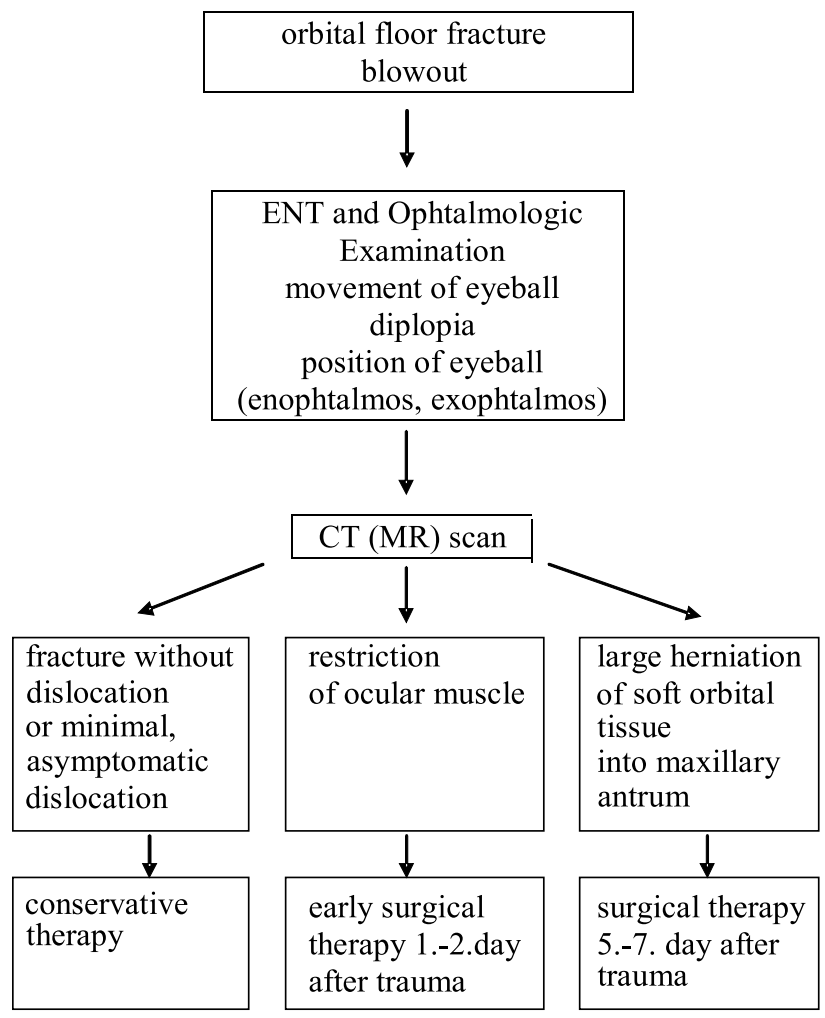


fore, the prognosis for eyeball movement recovery after a surgical reduction or a conservative therapy is significantly worse in children than in adults.

The second unique feature of orbital floor fractures in children concerns the surgical approach used for fracture reduction, as permanent dentition interferes with the transantral approach. This is the reason why a transconjunctival or a subciliar incision is selected in children.

The final issue concerns the selection of materials for reconstruction of the orbital floor in cases where bony fragments cannot be impacted after a reduction. No consensus exists on the choice of implants for orbital floor reconstruction. The literature mentions different materials $(1,3$, $4,6,7,8,10,13$ ) such as autogenous (bone, periosteum, fascia, cartilage), heterologous (lyophilized dura mater, pericardium, bovine collagenous fibres, lyophilized cartilage), or resorbable (Ethisorb, gelfilm, polylactic and polyglycolic acid plates and screws) and nonresorbable synthetic materials (teflon, silicone, silastic, collagen, titanium mesh).

Pelvicol is considered as the only type of absorbable material that is suitable for use in ENT surgery and in ophthalmosurgery with a very low potential for future biohazard. Collaboration with tissue banks does not have any disadvantages, and the cost of Pelvicol compared to the cost of human tissues is relative low. Cheung et al. described clinical features, treatment, and histological changes in a patient with severe localized orbital inflammation associated with the use of a porcine dermal collagen xenograft (Permacol) as an orbital floor implant in a 14-year-old boy (4).

In the presented case, Pelvicol was selected as a natural xenogeneic tissue (porcine dermis) ready for use. Pelvicol, which is usually used in urogenital surgery, was implanted by ophthalmologists in scleroplastic surgery without a rejection, sensitisation, irritancy, or an allergic reaction. We demonstrate the first using of Pelvicol for orbital floor fracture in literature. Pelvicol is an acellular dermal collagen with a portion composed of elastic fibres. Cross-linking of collagen improves resistance against collagenases; therefore, it does not induce an immune response and it provides a matrix for ingrowth of new tissue.

The authors believe that the above mentioned characteristics of Pelvicol and its acceptable flexibility, natural role in the body, and durability are advantageous properties for ophthalmologic and ENT plastic surgery.

Table 1 presents diagnostic and therapeutic procedures in orbital floor fractures in children.

\section{References}

1. Büchel P, Rabal A, Seto I, Iizuka T. Reconstruction of orbital floor fracture with polyglactin 910/Polydioxanon patch (Ethisorb): a retrospective study. J Oral Maxillofac Surg 2005;63:646-50.

2. Cheung D, Brown L, Sampath R. Localized inferior orbital fibrosis associated with porcine dermal collagen xenograft orbital floor implant. Ophthal Plast Reconstr Surg 2005;21:249-51.

3. Chrobok V, Vokurka J, Čelakovský P, Růžička J, Juran J. Fractures of the orbit and their influence on the position of ocular globe and ocular movement. Head and Neck Diseases 1998;(1)5-12.

4. Egbert JE. Orbital trauma. Current Opininon Otolaryngol Head Neck Surg 1997; 5:228-33.

5. Eppley BL. Use of resorbable plates and screw in pediatric fractures. J Oral Maxillofac Surg 2005;63:383-91.

6. Iatrou I, Theologie-Lygidakis N, Angelopoulos A. Use of membrane and bone grafts in the reconstruction of orbital fractures. Oral Surg Oral Med Pathol Oral Radiol Endod 2001;91:281-6.

7. Lee $\mathrm{HH}$, Alcaraz N, Reino A, Lawson W. Reconstruction of orbital floor fractures with maxillary bone. Arch Otolaryngol Head Neck Surg 1998;124:56-9.

8. Lelli GJ Jr, Milite J,: Orbital floor fractures: evaluation, indications, approach, and pearls from an ophthalmologist's perspective. Facial Plast Surg 2007;23(3): 190-7.

9. Pellant A, Frank M, Juran J, Hořecky D, Jiroušek D. Frakturen der Mittel und Oberetage des Gesichts, verbunden mit Verletzungen der Orbita. Otorhinolaryngol Nova 1992;2:268-71.

10. Raz S. Gelfilm and blowout fractures. J Laryngol Otol 1976;90:699-02.

11. Rowe-Jones JM, Adam EJ, Moore-Gillon V: Subtle diagnostic markers of orbital floor blow-out fracture on coronal CT scan. J Laryngol Otol 1993;107:161-2.

12. Saunders CJ, Whetzel TP, Stokes RB, Wong GB, Stevenson TR. Transantral endoscopic orbital floor exploration: a cadaver and clinical study. Plast Reconstr Surg 1997; 100:575-81.

13. Scapini DA, Mathog RH. Repair of orbital floor fractures with Marlex mesh. Laryngoscope 1989;99:697-701.

Submitted November 2008. Accepted December 2008 .

\section{Corresponding author:}

Viktor Chrobok M.D., Ph.D., Department of Otorhinolaryngology and Head and Neck Surgery, Regional Hospital of Pardubice, Kyjevská 44, 53203 Pardubice, Czech Republic; e-mail: viktor.chrobok@nemocnice-pardubice.cz 